\title{
THE ARNOLDI PROCESS AND GMRES FOR NEARLY SYMMETRIC MATRICES*
}

\author{
BERNHARD BECKERMANN ${ }^{\dagger}$ AND LOTHAR REICHEL ${ }^{\ddagger}$
}

\begin{abstract}
Matrices with a skew-symmetric part of low rank arise in many applications, including path following methods and integral equations. This paper explores the properties of the Arnoldi process when applied to such a matrix. We show that an orthogonal Krylov subspace basis can be generated with short recursion formulas and that the Hessenberg matrix generated by the Arnoldi process has a structure, which makes it possible to derive a progressive GMRES method. Eigenvalue computation is also considered.
\end{abstract}

Key words. low-rank perturbation, iterative method, solution of linear system, eigenvalue computation

AMS subject classifications. $65 \mathrm{~F} 10,65 \mathrm{~F} 15$

DOI. $10.1137 / 060668274$

1. Introduction. This paper discusses the Arnoldi process applied to a large matrix $A \in \mathbb{R}^{n \times n}$ with a skew-symmetric part

$$
A-A^{*}=\sum_{k=1}^{s} f_{k} g_{k}^{*}, \quad f_{k}, g_{k} \in \mathbb{R}^{n}
$$

of low rank $s$. In particular, we assume that $s \ll n$. The superscript $*$ denotes transposition and, when applicable, complex conjugation. We present our results for matrices $A$ and vectors $f_{k}$ and $g_{k}$ with real entries; however, our algorithms also can be applied to matrices and vectors with complex entries.

Linear systems of equations

$$
A x=b
$$

with large matrices of this kind arise in path following methods, from integral equations as well as from certain boundary value problems for partial differential equations.

The generalized minimal residual (GMRES) method is one of the most popular iterative methods for the solution of large linear systems of equations with a nonsymmetric matrix. The standard implementation of GMRES is based on the Arnoldi process; see, e.g., Saad [15, section 6.5]. Application of $j$ steps of the Arnoldi process to the matrix $A$ with initial vector $r_{0} \neq 0$ yields the decomposition

$$
A V_{j}=V_{j} H_{j}+h_{j} e_{j}^{*}
$$

where $V_{j}=\left[v_{1}, v_{2}, \ldots, v_{j}\right] \in \mathbb{R}^{n \times j}$ and $h_{j} \in \mathbb{R}^{n}$ satisfy $V_{j}^{*} V_{j}=I_{j}, V_{j}^{*} h_{j}=0$, and $v_{1}=r_{0} /\left\|r_{0}\right\|$. Moreover, $H_{j} \in \mathbb{R}^{j \times j}$ is an upper Hessenberg matrix. Throughout

*Received by the editors August 24, 2006; accepted for publication (in revised form) by M. Benzi July 2, 2007; published electronically February 6, 2008.

http://www.siam.org/journals/simax/30-1/66827.html

${ }^{\dagger}$ Laboratoire Painlevé UMR 8524 (ANO-EDP), UFR Mathématiques - M3, UST Lille, F-59655 Villeneuve d'Ascq CEDEX, France (bbecker@math.univ-lille1.fr). This author's research was supported in part by INTAS research network NeCCA 03-51-6637.

${ }^{\ddagger}$ Department of Mathematical Sciences, Kent State University, Kent, OH 44242 (reichel@math. kent.edu). This author's research was supported in part by NSF grant DMS-0107858 and an OBR Research Challenge Grant. 
this paper $I_{j}$ denotes the identity matrix of order $j, e_{k}$ denotes the $k$ th column of an identity matrix of appropriate order, and $\|\cdot\|$ denotes the Euclidean vector norm. For ease of discussion, we will assume that $j$ is small enough so that the decomposition (1.3) with the stated properties exists.

When $h_{j} \neq 0$, we can express (1.3) in the form

$$
A V_{j}=V_{j+1} \bar{H}_{j}
$$

where $v_{j+1}=h_{j} /\left\|h_{j}\right\|$ and

$$
V_{j+1}=\left[V_{j}, v_{j+1}\right] \in \mathbb{R}^{n \times(j+1)}, \quad \bar{H}_{j}=\left[\begin{array}{c}
H_{j} \\
\left\|h_{j}\right\| e_{j}^{*}
\end{array}\right] \in \mathbb{R}^{(j+1) \times j} .
$$

The computation of the Arnoldi decompositions (1.3) or (1.4) of a general $n \times n$ matrix $A$ requires the evaluation of $j$ matrix-vector products with $A$ and of about $j^{2} / 2$ inner products with $n$-vectors. The latter demands $\mathcal{O}\left(n j^{2}\right)$ arithmetic floating point operations (flops) and may dominate the computational work. The Arnoldi process determines the columns of $V_{j}$ in order and requires access to all the previously generated columns to compute the next one; in particular, all the columns of $V_{j}$ have to be stored; see, e.g., Saad [15, section 6.3] for a thorough treatment of the Arnoldi process. Computation of the $j$ th iterate by GMRES also requires the whole matrix $V_{j}$ to be available. To limit the demand of computer memory, GMRES is often restarted periodically, say, every $m$ steps. This restarted GMRES method is denoted by GMRES $(m)$. Restarting may reduce the rate of convergence of GMRES significantly.

In section 2, we show that the property (1.1) of $A$ makes it possible to determine the columns $v_{k}$ of $V_{j}$ with a short recursion formula, the number of terms of which depends on $s$ in (1.1) but can be bounded independently of $k$. The recursion formula allows the computation of all the columns of $V_{j}$ in only $\mathcal{O}(n j)$ flops. Moreover, the computation of $v_{k}$ for large $k$ does not require access to all the previously computed columns of $V_{j}$. Section 3 discusses the structure of the Hessenberg matrix $H_{j}$ in (1.3) when $A$ satisfies (1.1) and presents a fast algorithm for determining the Arnoldi decomposition (1.4).

The short recursion formula for the columns of $V_{j}$ and the structure of $H_{j}$ make it possible to derive a progressive GMRES method for the solution of linear systems (1.2) with a matrix that satisfies (1.1). Such a method is described in section 4 . The storage requirement of the method, as well as the computational effort per iteration, are bounded independently of the number of iterations $j$. This makes it possible to apply the method without periodic restarts. Computed examples are presented in section 5 and concluding remarks can be found in section 6 .

Recently, Barth and Manteuffel [4] presented iterative methods of conjugate gradient type for linear systems of equations of the kind considered in the present paper. Specifically, they considered linear systems of equations with a generalized $B$-normal $(\ell, m)$ matrix. This type of matrix is characterized by the existence of polynomials $p_{\ell}$ and $q_{m}$ of degrees $\ell$ and $m$, respectively, such that the matrix

$$
A^{\dagger} q_{m}(A)-p_{\ell}(A)
$$

is of low rank, where $A^{\dagger}=B^{-1} A^{*} B$ and $B$ is a Hermitian positive definite matrix. The matrix $A^{\dagger}$ is the adjoint of $A$ with respect to the $B$-inner product

$$
\langle u, v\rangle_{B}=u^{*} B v .
$$


In the terminology of Barth and Manteuffel [4] matrices $A$ that satisfy (1.1) are generalized $I$-normal $(1,0)$ matrices.

Barth and Manteuffel [4] derived their methods by generalizing the recurrence relations for orthogonal polynomials on the unit circle. The latter type of recurrence relations had previously been applied to iterative methods in [11, 12]; see also Arnold et al. [2] for a recent application to QCD computations. The derivation of our iterative methods for (1.2) differs from the derivation by Barth and Manteuffel [4] of their schemes in that we do not apply properties of orthogonal polynomials on the unit circle. Iterative methods for linear systems of equations with a matrix, whose symmetric part is positive definite and easily invertible, are described by Concus and Golub [7] and Widlund [18].

2. Generation of an orthogonal Krylov subspace basis. Introduce the Krylov subspace

$$
\mathcal{K}_{j}(A, b)=\operatorname{span}\left\{b, A b, A^{2} b, \ldots, A^{j-1} b\right\},
$$

which we assume to be of dimension $j$. The columns of the matrix $V_{j}$ in (1.3) form an orthonormal basis of $\mathcal{K}_{j}(A, b)$.

Let $f_{k}$ and $g_{k}$ be the vectors in (1.1) and define the matrices

$$
F=\left[f_{1}, f_{2}, \ldots, f_{s}\right], \quad G=\left[g_{1}, g_{2}, \ldots, g_{s}\right],
$$

which we may assume to be of full rank; otherwise we can reduce $s$. We express (1.1) as

$$
A-A^{*}=F G^{*}
$$

and note that

$$
F G^{*}=-G F^{*}
$$

It follows from (2.4) and the fact that $F$ and $G$ are of full rank that $s$ is even and that there is a unique matrix $C \in \mathbb{R}^{s \times s}$, such that

$$
G=F C .
$$

The fact that $s$ is even can be seen by substituting (2.5) into (2.4). This yields $C^{*}=-C$. Therefore, when $s$ is odd, $C$ is singular and $G$ is not of full rank. Use of the representation (2.5) of $G$ reduces the computational work in the algorithms presented in sections 3 and 4 .

Example 2.1. In many applications that involve a matrix $A$ with a skew-symmetric part of low rank, the matrix is given in the form

$$
A=M+\sum_{k=1}^{s / 2} f_{k} g_{k}^{*}
$$

with $M \in \mathbb{R}^{n \times n}$ symmetric. Then (1.1) can be expressed as

$$
A-A^{*}=\sum_{k=1}^{s / 2} f_{k} g_{k}^{*}-\sum_{k=1}^{s / 2} g_{k} f_{k}^{*}
$$

and we may choose

$$
F=\left[f_{1}, f_{2}, \ldots, f_{s / 2}, g_{1}, g_{2}, \ldots, g_{s / 2}\right], \quad C=\left[\begin{array}{cc}
0 & -I_{s / 2} \\
I_{s / 2} & 0
\end{array}\right] .
$$


Introduce the vectors

$$
f_{\ell, k}=V_{k} V_{k}^{*} f_{\ell}, \quad 1 \leq \ell \leq s, \quad 1 \leq k \leq j .
$$

Then

$$
f_{\ell, k} \in \mathcal{K}_{k}\left(A, r_{0}\right), \quad f_{\ell, k}-f_{\ell} \perp \mathcal{K}_{k}\left(A, r_{0}\right) .
$$

Moreover, for each $\ell$, the $f_{\ell, k}$ satisfy the recursion

$$
\left\{\begin{array}{l}
f_{\ell, k}=f_{\ell, k-1}+v_{k} v_{k}^{*} f_{\ell}, \quad k=2,3, \ldots, j, \\
f_{\ell, 1}=v_{1} v_{1}^{*} f_{\ell}
\end{array}\right.
$$

Let

$$
v_{k}^{\prime}=A^{*} v_{k}+\sum_{\ell=1}^{s} g_{\ell}^{*} v_{k}\left(f_{\ell}-f_{\ell, k}\right)
$$

Then (1.1) gives

$$
v_{k}^{\prime}-A v_{k}=\sum_{\ell=1}^{s} g_{\ell}^{*} v_{k}\left(f_{\ell}-f_{\ell, k}\right)-\left(A-A^{*}\right) v_{k}=-\sum_{\ell=1}^{s} g_{\ell}^{*} v_{k} f_{\ell, k} .
$$

We may assume that $A v_{k} \notin \mathcal{K}_{k}\left(A, r_{0}\right)$, because otherwise range $\left(V_{k}\right)$ is an invariant subspace of $A$, which contains the solution of the linear system (1.2); see, e.g., Saad [15, section 6.5.4] for details. The following properties of $v_{k}^{\prime}$ are a consequence of the above discussion.

Proposition 2.2. Let $v_{k}^{\prime}$ be defined by (2.9) and assume that $\operatorname{dim} \mathcal{K}_{k+1}\left(A, r_{0}\right)=$ $k+1$. Then

$$
v_{k}^{\prime} \in \mathcal{K}_{k+1}\left(A, r_{0}\right) \backslash \mathcal{K}_{k}\left(A, r_{0}\right), \quad v_{k}^{\prime} \perp \mathcal{K}_{k-2}\left(A, r_{0}\right) .
$$

Proof. The requirement that $\mathcal{K}_{k+1}\left(A, r_{0}\right)$ be of dimension $k+1$ secures that $A v_{k} \notin \mathcal{K}_{k}\left(A, r_{0}\right)$. Equation (2.7) yields that $v_{k}^{\prime}-A v_{k} \in \mathcal{K}_{k}\left(A, r_{0}\right)$, and this establishes the left-hand side of (2.11).

It follows from the Arnoldi decomposition (1.3) that $v_{k} \perp A v_{\ell}$ for $1 \leq \ell \leq k-2$, or, equivalently, that $A^{*} v_{k} \perp v_{\ell}$ for $1 \leq \ell \leq k-2$. The latter property, in combination with (2.7) and (2.9), shows the orthogonality relation (2.11).

Equation (2.10) yields the expression

$$
v_{k}^{\prime}=A v_{k}-\sum_{\ell=1}^{s} g_{\ell}^{*} v_{k} f_{\ell, k}
$$

which we use to evaluate $v_{k}^{\prime}$. Orthogonalization against the vectors $v_{k-1}$ and $v_{k}$, and normalization of the resulting vector, gives the Arnoldi vector $v_{k+1}$. In what follows we will write this operation more explicitly as

$$
v_{k}^{\prime}=t_{k+1, k} v_{k+1}+t_{k, k} v_{k}+t_{k-1, k} v_{k-1}, \quad k \geq 1,
$$

where

$$
t_{k-1, k}=v_{k-1}^{*} v_{k}^{\prime}, \quad t_{k, k}=v_{k}^{*} v_{k}^{\prime}, \quad t_{k+1, k}=v_{k+1}^{*} v_{k}^{\prime},
$$

with $v_{0}=0$ and $t_{k+1, k}=\left\|v_{k}^{\prime}-t_{k, k} v_{k}-t_{k, k-1} v_{k-1}\right\|>0$. The computations for generating the orthogonal Krylov subspace basis, and for determining the matrix $\bar{H}_{j}$ in (1.4), are summarized in Algorithm 3.2 of the following section.

Copyright (C) by SIAM. Unauthorized reproduction of this article is prohibited. 
3. Structure of the Hessenberg matrices. This section discusses the structure of the matrices $H_{j}=\left[h_{k, \ell}\right]$ and $\bar{H}_{j}=\left[h_{k, \ell}\right]$ in the Arnoldi decompositions (1.3) and (1.4), respectively. It is convenient to introduce the following terminology. For an integer $m$, the $m$-diagonal of a matrix $B=\left[b_{k, \ell}\right]$ consists of all entries of the form $b_{k, k+m}$. The $m$-upper ( $m$-lower) triangular part of $B$ is the submatrix comprising all entries on and above (below) the $m$-diagonal. For instance, the upper Hessenberg matrices $H_{j}$ and $\bar{H}_{j}$ have vanishing $(-2)$-lower triangular parts. Note that the $(-2)$ upper triangular part is not triangular.

Proposition 3.1. Let $\hat{F}_{j}=V_{j}^{*} F$ and $\hat{G}_{j}=V_{j}^{*} G$, where $F$ and $G$ are the matrices in (2.3) and $j \geq s$. Then the upper Hessenberg matrix $H_{j}$ in the Arnoldi decomposition (1.3) satisfies

$$
H_{j}-H_{j}^{*}=\hat{F}_{j} \hat{G}_{j}^{*}, \quad \hat{G}_{j} \hat{F}_{j}^{*}=-\hat{F}_{j} \hat{G}_{j}^{*},
$$

i.e., $H_{j}$ has a skew-symmetric part of rank s. Moreover, $H_{j}$ and $\hat{F}_{j} \hat{G}_{j}^{*}$ have the same 2-upper triangular parts.

Proof. It follows from (1.3) and (2.3) that

$$
H_{j}=V_{j}^{*} A V_{j}=V_{j}^{*}\left(A^{*}+F G^{*}\right) V_{j}=H_{j}^{*}+\hat{F}_{j} \hat{G}_{j}^{*},
$$

which shows (3.1). Since the $(-2)$-lower triangular part of $H_{j}$ vanishes, (3.1) yields the 2-upper triangular part of $H_{j}$.

The proposition shows that $H_{j}$ is an order- $(1, s+1)$ quasi-separable matrix; see, e.g., Eidelman, Gohberg, and Olshevsky [9] for a recent discussion on this kind of matrix.

We turn to the entries in the tridiagonal part of $\bar{H}_{j}$. In accordance with (2.14), we define the matrix $\bar{T}_{j}=\left[t_{m, k}\right] \in \mathbb{R}^{(j+1) \times j}$ with entries $t_{m, k}=v_{m}^{*} v_{k}^{\prime}$. Notice that $\bar{T}_{j}$ is tridiagonal by Proposition 2.2. Substitution of (2.6) into (2.12) gives

$$
v_{k}^{\prime}=A v_{k}-\sum_{\ell=1}^{s}\left(g_{\ell}^{*} v_{k}\right) V_{k} V_{k}^{*} f_{\ell}=A v_{k}-V_{k} V_{k}^{*} F G^{*} v_{k}=A v_{k}-V_{k} \hat{F}_{k} \hat{G}_{k}^{*} e_{k},
$$

and, taking into account that $e_{m}^{*} \hat{F}_{k} \hat{G}_{k}^{*} e_{k}=e_{m}^{*} \hat{F}_{j} \hat{G}_{j}^{*} e_{k}$ for $m \leq k \leq j$, we get for the entries $h_{m, k}=v_{m}^{*} A v_{k}$ of $\bar{H}_{j}$ the formula

$$
h_{m, k}= \begin{cases}t_{k+1, k}, & m=k+1, \\ t_{m, k}+e_{m}^{*} \hat{F}_{j} \hat{G}_{j}^{*} e_{k}, & k-1 \leq m \leq k, \\ e_{m}^{*} \hat{F}_{j} \hat{G}_{j}^{*} e_{k}, & 1 \leq m<k-1 .\end{cases}
$$

Thus, the matrix $\hat{F}_{j} \hat{G}_{j}^{*}$ contributes to the upper triangular part of $\bar{H}_{j}$, and the matrix $\bar{T}_{j}$, which expresses the orthogonalization of the vectors $v_{k}^{\prime}$, contributes to the tridiagonal part; in MATLAB notation, we have

$$
\bar{H}_{j}=\bar{T}_{j}+\operatorname{triu}\left(\hat{F}_{j} \hat{G}_{j}^{*}, 0\right) .
$$

Combining (2.9) with (2.7) yields

$$
v_{m}^{*} v_{k}^{\prime}=v_{m}^{*} A^{*} v_{k}=\left(v_{k}^{*} A v_{m}\right)^{*}, \quad 1 \leq m \leq k,
$$

and comparison with (2.14) gives

$$
t_{k-1, k}=t_{k, k-1}>0, \quad t_{k, k}=h_{k, k}^{*} .
$$


We describe an algorithm for the computation of the matrices $\bar{H}_{j}$ and $V_{j+1}$ in the decomposition (1.4), assuming that the decomposition exists. The matrix $\bar{H}_{j}$ is represented in decomposed form (3.2) by the matrices $\hat{F}_{j}, \hat{G}_{j}$, and $\bar{T}_{j}$, which in the algorithm are represented without subscript $j$. The subscripts used in the algorithm denote row and column indices. Thus, $\hat{F}_{k, \text { : }}$ denotes the $k$ th row of the matrix $\hat{F}_{j}$. At iteration $k$, we let $\tilde{F}=\left[f_{1, k}, f_{2, k}, \ldots, f_{s, k}\right]$.

Algorithm 3.2. Generation of the matrices $\bar{H}_{j}$ and $V_{j+1}$.

Input: $A \in \mathbb{R}^{n \times n}, F=\left[f_{1}, f_{2}, \ldots, f_{s}\right], G=\left[g_{1}, g_{2}, \ldots, g_{s}\right] \in \mathbb{R}^{n \times s}, r_{0} \in \mathbb{R}^{n}, j$;

Output: $\bar{T}=\left[t_{\ell, k}\right] \in \mathbb{R}^{(j+1) \times j}, \hat{F}, \hat{G} \in \mathbb{R}^{(j+1) \times s}, V_{j+1}=\left[v_{1}, v_{2}, \ldots, v_{j+1}\right] \in$ $\mathbb{R}^{n \times(j+1)}$;

1. $\tilde{F}:=0$

2. $v_{1}:=r_{0} /\left\|r_{0}\right\|$;

3. for $k=1: j$

4. $\hat{F}_{k,:}:=v_{k}^{*} F ; \hat{G}_{k,:}:=v_{k}^{*} G$;

5. $\tilde{F}:=\tilde{F}+v_{k} \hat{F}_{k,:}$;

6. $v^{\prime}:=A v_{k}-\tilde{F} \hat{G}_{k,:}^{*}$;

7. if $k>1$ then

8. $t_{k-1, k}:=v_{k-1}^{*} v^{\prime} ; v^{\prime}:=v^{\prime}-t_{k-1, k} v_{k-1}$;

9. endif

10. $t_{k, k}:=v_{k}^{*} v^{\prime} ; v^{\prime}:=v^{\prime}-t_{k, k} v_{k} ; t_{k+1, k}:=\left\|v^{\prime}\right\| ; v_{k+1}:=v^{\prime} / t_{k+1, k}$

11. endfor

We note that the computational effort of line 4 of the algorithm can be essentially halved by using the representation (2.5) of $G$.

Algorithm 3.2 can be applied to compute approximations of a few extreme eigenvalues and associated eigenvectors of $A$ similarly to the standard implementation of the Arnoldi process. Certain eigenvalues of $H_{j}$ are used to approximate selected eigenvalues of $A$. The structure of $H_{j}$ therefore is of interest.

Remark 3.3. Given a unitary matrix $Q \in \mathbb{C}^{j \times j}$, it follows from Proposition 3.1 that for the matrix

$$
S=Q^{*} H_{j} Q
$$

we have $\Sigma:=S-S^{*}=Q^{*} \hat{F}_{j} \hat{G}_{j}^{*} Q$, i.e., $S$ has a skew-symmetric part of rank $s$. If $S$ has an additional sparsity structure, then we may derive results similarly to Proposition 3.1. For instance, the matrix $S$ in the Schur normal form of $H_{j}$ is upper triangular, and thus $S$ may be written as a diagonal matrix plus the 1-upper triangular part of the matrix $\Sigma$. Similarly, the matrix $S$ obtained after one step of the QR-algorithm is upper Hessenberg and therefore may be written as a tridiagonal matrix plus the 2-upper triangular part of the matrix $\Sigma$.

We recall that in the QR-algorithm for eigenvalue computations the unitary factor $Q$ is chosen such that $R=Q^{*} H_{j}$ is upper triangular.

Remark 3.4. Consider the QR-decomposition $H_{j}=Q R$ with orthogonal $Q$ and upper triangular $R$. Here also the matrix $R$ has a structure: since $Q^{*}$ is known to be of lower Hessenberg form (see, e.g., the considerations of the next section), we see from Proposition 3.1 that the 3-upper triangular part of $Q^{*}\left(H_{j}-\hat{F}_{j} \hat{G}_{j}\right)$ contains only zeros, or, in other words, the 3-upper triangular parts of $R_{j}$ and of the matrix $Q^{*} \hat{F}_{j} \hat{G}_{j}$ of rank $s$ coincide.

The structure makes it possible to compute the matrix $R$ in $\mathcal{O}(j)$ flops, by representing $H_{j}$ in terms of the tridiagonal part of $H_{j}$ and the matrices $\hat{F}_{j}$ and $\hat{G}_{j}$, and by representing $R$ in terms of its $0-, 1$-, and 2-diagonals and the matrices $Q^{*} \hat{F}_{j}$ and $\hat{G}_{j}$. 
Since the computation of $R$ does not play a role in subsequent considerations, we omit the details.

4. A progressive GMRES algorithm. Let $x_{0} \in \mathbb{R}^{n}$ be an approximate solution of (1.2). GMRES determines a new approximate solution $x_{j}$ of (1.2), such that

$$
\left\|A x_{j}-b\right\|=\min _{x \in x_{0}+\mathcal{K}_{j}\left(A, r_{0}\right)}\|A x-b\|, \quad x_{j} \in x_{0}+\mathcal{K}_{j}\left(A, r_{0}\right) .
$$

The standard implementation of GMRES determines a correction of $x_{0}$, i.e., $x_{j}=$ $x_{0}+V_{j} x_{j}$, by substituting the decomposition (1.4) with $r_{0}=b-A x_{0}$ into (4.1); see, e.g., Saad [15, section 6.5] for details. This gives the equivalent minimization problem

$$
\min _{y \in \mathbb{R}^{j}}\left\|\bar{H}_{j} y-e_{1}\right\| r_{0}\|\|
$$

with solution $y_{j} \in \mathbb{R}^{j}$.

We solve the least-squares problem (4.2) by using the QR-factorization $\bar{H}_{j}=$ $Q_{j+1} \bar{R}_{j}$, where $Q_{j+1} \in \mathbb{R}^{(j+1) \times(j+1)}$ is orthogonal (or unitary in the case of complex $A, b)$ and

$$
\bar{R}_{j}=\left[\begin{array}{c}
R_{j} \\
0
\end{array}\right] \in \mathbb{R}^{(j+1) \times j},
$$

with $R_{j} \in \mathbb{R}^{j \times j}$ upper triangular. Let us first recall in the following paragraph and Proposition 4.1 the well-known construction of a QR-decomposition of the upper Hessenberg matrix $\bar{H}_{j}$ for a general matrix $A$. Subsequently, we explain in Proposition 4.2 how the structure of the matrix $A$ helps us to derive a progressive form of GMRES.

Following Saad [15, Chapter 6.5.3], we determine the matrix $Q_{j+1}$ by applying a product of Givens rotations to $\bar{H}_{j}$. Let $Q_{1}=[1]$ and define, for $k=1,2, \ldots, j$,

$$
Q_{k+1}^{*}=\Omega_{k+1}\left[\begin{array}{cc}
Q_{k}^{*} & 0 \\
0 & 1
\end{array}\right], \quad \Omega_{k+1}=\left[\begin{array}{ccc}
I_{k-1} & 0 & 0 \\
0 & c_{k}^{*} & s_{k} \\
0 & -s_{k} & c_{k}
\end{array}\right],
$$

with $s_{k} \geq 0$ and $s_{k}^{2}+\left|c_{k}\right|^{2}=1$ such that $\Omega_{k+1}$ is unitary (and reduces to a classical Givens rotation in the case of real data). Using the nested structure of $\bar{H}_{j}=\left[h_{k, \ell}\right]$, i.e., the fact that $\bar{H}_{j-1}$ is the leading $j \times(j-1)$ principal submatrix of $\bar{H}_{j}$, yields

$$
Q_{j+1}^{*} \bar{H}_{j}=\Omega_{j+1}\left[\begin{array}{cc}
Q_{j}^{*} \bar{H}_{j-1} & Q_{j}^{*} H_{j} e_{j} \\
0 & h_{j+1, j}
\end{array}\right]=\Omega_{j+1}\left[\begin{array}{cc}
R_{j-1} & * \\
0 & \tau_{j} \\
0 & h_{j+1, j}
\end{array}\right],
$$

with

$$
\tau_{j}=e_{j}^{*} Q_{j}^{*} H_{j} e_{j}
$$

Since multiplication by $\Omega_{j+1}$ affects only the last two rows, the matrices $R_{j}$ and $\bar{R}_{j}$ also have a nested substructure:

$$
\bar{R}_{j}=\left[\begin{array}{cc}
\bar{R}_{j-1} & * \\
0 & 0
\end{array}\right], \quad R_{j}=\left[\begin{array}{cc}
R_{j-1} & * \\
0 & *
\end{array}\right] .
$$

Copyright (c) by SIAM. Unauthorized reproduction of this article is prohibited. 
We have the following formulas for the coefficients $c_{j}, s_{j}$ of $\Omega_{j+1}$ and for the entries of $Q_{j+1}^{*}$.

Proposition 4.1. There holds

$$
s_{j}=\frac{t_{j+1, j}}{\sqrt{t_{j+1, j}^{2}+\left|\tau_{j}\right|^{2}}} \geq 0, \quad c_{j}=\frac{\tau_{j}}{\sqrt{t_{j+1, j}^{2}+\left|\tau_{j}\right|^{2}}},
$$

where $t_{j+1, j}=h_{j+1, j}$ is the last subdiagonal entry of $\bar{H}_{j}$ and $\tau_{j}$ is given by (4.5). The first $j$ rows of $Q_{j+1}^{*}$ are obtained by padding a zero on the right-hand side of the corresponding rows of $Q_{j}^{*}$. In particular, $Q_{j+1}^{*}$ is of lower Hessenberg form, with its lower triangular part coinciding with the lower triangular part of a rank-one matrix. Moreover, for $j \geq 3$,

$$
e_{j+1}^{*} Q_{j+1}^{*}=\left[-s_{j} e_{j}^{*} Q_{j}^{*}, c_{j}\right]=\left[*, s_{j} s_{j-1} c_{j-2},-s_{j} c_{j-1}, c_{j}\right] .
$$

Proof. The proof is obtained by direct calculations.

We are in a position to describe a progressive recurrence relation for the GMRES residual $r_{j}$, a simplified recurrence for its norm, as well as a simplified expression for the quantity $\tau_{j}$ defined by (4.5). In particular, the progressive GMRES algorithm does not require the entries of the matrices $R_{j}, \bar{H}_{j}$, and $Q_{j+1}$. Only the $c_{k}, s_{k}$ of the Givens rotations (4.4) and the quantities occurring in the recurrence relation for the Arnoldi vectors $v_{k}$ are needed.

Proposition 4.2. Let $r_{j}$ denote the residual vector associated with $x_{j}$, i.e.,

$$
r_{j}=b-A x_{j},
$$

and define recursively

$$
\gamma_{j}=-s_{j} \gamma_{j-1}, \quad j \geq 1,
$$

where $\gamma_{0}=\left\|r_{0}\right\|$. Then $\gamma_{j}=(-1)^{j}\left\|r_{j}\right\|$. Moreover,

$$
r_{j}=s_{j}^{2} r_{j-1}+\gamma_{j} c_{j}^{*} v_{j+1}, \quad j \geq 1 .
$$

Finally, define the vectors $p_{j} \in \mathbb{R}^{s}$ recursively by

$$
p_{j}^{*}=-s_{j-1} p_{j-1}^{*}+c_{j-1} e_{j}^{*} \hat{F}_{j}, \quad j \geq 2,
$$

and $p_{1}^{*}=\hat{F}_{1}$. Then we get for the scalar $\tau_{j}$ defined by (4.5) the expression

$$
\tau_{j}=c_{j-1} t_{j, j}-s_{j-1} c_{j-2} t_{j-1, j}+p_{j}^{*} \hat{G}_{j}^{*} e_{j}, \quad j \geq 2,
$$

with $c_{0}=1$ and $\tau_{1}=t_{1,1}^{*}$.

Proof. We start by establishing the formula

$$
r_{j}=\gamma_{j} V_{j+1} Q_{j+1} e_{j+1} .
$$

A different proof is presented by Saad [15, Proposition 6.9]. From the definition of GMRES, we have that $r_{j}=P_{A \mathcal{K}_{j}\left(A, r_{0}\right)}^{\perp} r_{0}$, where $P_{A \mathcal{K}_{j}\left(A, r_{0}\right)}$ denotes the orthogonal projector onto $A \mathcal{K}_{j}\left(A, r_{0}\right)$ and $P_{A \mathcal{K}_{j}\left(A, r_{0}\right)}^{\perp}=I-P_{A \mathcal{K}_{j}\left(A, r_{0}\right)}$ denotes the orthogonal projector onto the complement. Denote by $\bar{Q}_{j} \in \mathbb{R}^{(j+1) \times j}$ the matrix made up of the first $j$ columns of $Q_{j+1}$. From (1.4) and (4.3), we obtain that 
$A V_{j}=V_{j+1} Q_{j+1} \bar{R}_{j}=V_{j+1} \bar{Q}_{j} R_{j}$. Since $R_{j}$ is invertible, we see that an orthonormal basis of $A \mathcal{K}_{j}\left(A, r_{0}\right)$ is given by the columns of $V_{j+1} \bar{Q}_{j}$, implying that

$$
\begin{aligned}
r_{j} & =r_{0}-P_{A \mathcal{K}_{j}\left(A, r_{0}\right)} r_{0}=V_{j+1} Q_{j+1} Q_{j+1}^{*} V_{j+1}^{*} r_{0}-V_{j+1} \bar{Q}_{j} \bar{Q}_{j}^{*} V_{j+1}^{*} r_{0} \\
& =V_{j+1}\left(Q_{j+1} Q_{j+1}^{*}-\bar{Q}_{j} \bar{Q}_{j}^{*}\right) e_{1}\left\|r_{0}\right\|=V_{j+1} Q_{j+1} e_{j+1} e_{j+1}^{*} Q_{j+1}^{*} e_{1}\left\|r_{0}\right\| .
\end{aligned}
$$

It follows from (4.7) and (4.9) that

$$
\gamma_{0} e_{j+1}^{*} Q_{j+1}^{*} e_{1}=\gamma_{0}\left(-s_{j}\right) e_{j}^{*} Q_{j}^{*} e_{1}=\cdots=\gamma_{0}\left(-s_{j}\right)\left(-s_{j-1}\right) \ldots\left(-s_{1}\right)=\gamma_{j} .
$$

This establishes (4.13). Since $V_{j+1} Q_{j+1}$ has orthonormal columns and $s_{k} \geq 0$ by Proposition 4.1, we may conclude by taking norms in (4.13) that $\left|\gamma_{j}\right|=\left\|r_{j}\right\|=(-1)^{j} \gamma_{j}$.

The updating formula (4.10) is now an immediate consequence of (4.13): by (4.7),

$$
r_{j}=\gamma_{j} V_{j+1}\left[-s_{j} e_{j}^{*} Q_{j}^{*}, c_{j}\right]^{*}=-s_{j} \frac{\gamma_{j}}{\gamma_{j-1}} r_{j-1}+\gamma_{j} c_{j}^{*} v_{j+1} .
$$

It remains to show (4.12). From (4.7) and (4.11) we conclude by recurrence on $j$ that

$$
p_{j}^{*}=e_{j}^{*} Q_{j}^{*} \hat{F}_{j}, \quad j \geq 1 .
$$

The structure of $H_{j}$, together with (4.7) and (4.13), yields for $j \geq 2$ that

$$
\begin{aligned}
\tau_{j} & =e_{j}^{*} Q_{j}^{*} H_{j} e_{j} \\
& =e_{j}^{*} Q_{j}^{*}\left(\left[\begin{array}{c}
0 \\
\vdots \\
0 \\
t_{j-1, j} \\
t_{j, j}
\end{array}\right]+\hat{F}_{j} \hat{G}_{j}^{*} e_{j}\right)=\left[-s_{j-1} c_{j-2}, c_{j-1}\right]\left[\begin{array}{c}
t_{j-1, j} \\
t_{j, j}
\end{array}\right]+p_{j}^{*} \hat{G}_{j}^{*} e_{j} .
\end{aligned}
$$

When $j=1$, we get by using $Q_{1}=[1]$ and (3.3) that $\tau_{1}=h_{1,1}=t_{1,1}^{*} \cdot \quad \square$

By applying a suitable linear operator $L$, such that $L r_{k}=x_{k}$ for $0 \leq k \leq j+1$, to the recurrence relation (4.10) of the residuals, we obtain an updating formula for the GMRES iterates in terms of the auxiliary vectors $z_{k}=L v_{k}$ and $w_{\ell, k}=L f_{\ell, k}$, which together with the recursive computation of these new vectors is described in the following proposition.

Proposition 4.3. Let $\operatorname{dim} \mathcal{K}_{j+1}\left(A, r_{0}\right)=j+1$ and define recursively

$$
\begin{aligned}
& w_{\ell, k}=w_{\ell, k-1}+v_{k}^{*} f_{\ell} z_{k}, \quad 0<k \leq j \\
& z_{k+1}=-\frac{1}{t_{k+1, k}}\left(v_{k}+t_{k, k} z_{k}+t_{k-1, k} z_{k-1}+\sum_{\ell=1}^{s} g_{\ell}^{*} v_{k} w_{\ell, k}\right), \quad 1<k \leq j,
\end{aligned}
$$

together with the initializations

$$
w_{\ell, 0}=0, \quad z_{1}=\frac{x_{0}}{\gamma_{0}}, \quad z_{2}=-\frac{1}{t_{2,1}}\left(v_{1}+t_{1,1}^{*} z_{1}\right) .
$$

Then we have for $0<k \leq j$ the updating formula

$$
x_{k}=s_{k}^{2} x_{k-1}+\gamma_{k} c_{k}^{*} z_{k+1} .
$$

Copyright $@$ by SIAM. Unauthorized reproduction of this article is prohibited. 
Proof. Consider the QR-factorization

$$
\left[r_{0}, A r_{0}, \ldots, A^{j} r_{0}\right]=V_{j+1} S_{j+1},
$$

i.e., $S_{j+1} \in \mathbb{R}^{(j+1) \times(j+1)}$ is upper triangular and invertible by assumption on $j$. The projector

$$
P=V_{j+1} S_{j+1}\left(I_{j+1}-e_{1} e_{1}^{*}\right) S_{j+1}^{-1} V_{j+1}^{*}
$$

satisfies

$$
P\left(\sum_{k=0}^{j} \alpha_{k} A^{k} r_{0}\right)=\sum_{k=1}^{j} \alpha_{k} A^{k} r_{0}, \quad(I-P)\left(\sum_{k=0}^{j} \alpha_{k} A^{k} r_{0}\right)=\alpha_{0} r_{0} .
$$

As a consequence, defining the linear operator $L$ by

$$
L v=\frac{r_{0}^{*}(I-P) v}{r_{0}^{*} r_{0}} x_{0}-A^{-1} P v
$$

we get for any $u \in \mathcal{K}_{j}\left(A, r_{0}\right)$ that

$$
L\left(b-A\left(x_{0}+u\right)\right)=x_{0}+u .
$$

In particular, we obtain $L r_{k}=x_{k}$ for $0 \leq k \leq j$, as claimed above. In order to see that the vectors $z_{k+1}$ and $w_{\ell, k}$ defined by

$$
z_{k+1}=L v_{k+1}, \quad w_{\ell, k}=L f_{\ell, k}, \quad 0 \leq k \leq j,
$$

can be computed via the relations (4.14)-(4.16), we argue by recurrence on $k$ : applying $L$ to the relations $f_{\ell, 0}=0, v_{1}=r_{0} / \gamma_{0}=\left(b-A x_{0}\right) / \gamma_{0}$, and $A v_{1}=h_{2,1} v_{2}+h_{1,1} v_{1}=$ $t_{2,1} v_{2}+t_{1,1}^{*} v_{1}$, respectively, leads to the initializations (4.16). Similarly, for (4.14) we apply $L$ to (2.8), and (4.15) is obtained by applying $L$ both to (2.12) and (2.13), where we notice that $L\left(A v_{k}\right)=-v_{k}$. Finally, the recurrence relation (4.17) for the GMRES iterates follows by applying $L$ to (4.10).

Let $W_{j}=\left[w_{1, j}, w_{2, j}, \ldots, w_{s, j}\right] \in \mathbb{R}^{n \times s}$. Then (4.14) can be written as

$$
W_{j}=W_{j-1}+z_{j} e_{j}^{*} \hat{F}_{j}, \quad W_{1}=\frac{x_{0}}{\gamma_{0}} \hat{F}_{1}
$$

and

$$
\sum_{\ell=1}^{s} g_{\ell}^{*} v_{j} w_{\ell, j}=W_{j} \hat{G}_{j}^{*} e_{j}
$$

Algorithm 4.4 below works with the matrices $W_{j}$ rather than with their columns individually. The notation of Algorithm 4.4 follows that of Algorithm 3.2. In particular, the matrices $W_{j}$ are stored in $W$.

Algorithm 4.4. Progressive GMRES.

Input: $A \in \mathbb{R}^{n \times n}, F=\left[f_{1}, f_{2}, \ldots, f_{s}\right], G=\left[g_{1}, g_{2}, \ldots, g_{s}\right] \in \mathbb{R}^{n \times s}, b, x_{0} \in \mathbb{R}^{n}$;

Output: GMRES iterates $x_{j} \in \mathbb{R}^{n}$;

$\%$ initialization

1. $r_{0}:=b-A x_{0} ; \gamma_{0}:=\left\|r_{0}\right\|$;

2. $v_{1}:=r_{0} / \gamma_{0} ; z_{1}:=x_{0} / \gamma_{0}$;

Copyright $@$ ㅇ by SIAM. Unauthorized reproduction of this article is prohibited. 
$\% j=1$

3. $\hat{F}_{1,:}:=v_{1}^{*} F ; \hat{G}_{1,:}:=v_{1}^{*} G$;

4. $p_{1}^{*}:=\hat{F}_{1,:} ; \tilde{F}:=v_{1} \hat{F}_{1,:} ; W:=x_{0} \hat{F}_{1} / \gamma_{0}$;

5. $v^{\prime}:=A v_{1}-\tilde{F} \hat{G}_{1,:}^{*}$;

6. $t_{1,1}:=v_{1}^{*} v^{\prime} ; v^{\prime}:=v^{\prime}-t_{1,1} v_{1}$

7. $t_{2,1}:=\left\|v^{\prime}\right\| ; v_{2}:=v^{\prime} / t_{2,1}$;

8. $\tau_{1}:=t_{1,1}^{*}$;

9. $c_{1}:=\tau_{1} /\left(\left|\tau_{1}\right|^{2}+t_{2,1}^{2}\right)^{1 / 2} ; s_{1}:=t_{2,1} /\left(\left|\tau_{1}\right|^{2}+t_{2,1}^{2}\right)^{1 / 2} ; \gamma_{1}:=-s_{1} \gamma_{0}$;

10. $z_{2}:=-\left(v_{1}+t_{1,1}^{*} z_{1}\right) / t_{2,1} ; x_{1}:=s_{1}^{2} x_{0}+\gamma_{1} c_{1}^{*} z_{2}$;

$\% j>1$

11. for $j=2,3, \ldots$ until convergence

12. $\hat{F}_{j,:}:=v_{j}^{*} F ; \hat{G}_{j,:}:=v_{j}^{*} G$;

13. $p_{j}^{*}:=-s_{j-1} p_{j-1}^{*}+c_{j-1} \hat{F}_{j,:} ; \tilde{F}:=\tilde{F}+v_{j} \hat{F}_{j,:} ; W:=W+z_{j} \hat{F}_{j,:} ;$

14. $v^{\prime}:=A v_{j}-\tilde{F} \hat{G}_{j,:}^{*}$

15. $t_{j-1, j}:=v_{j-1}^{*} v^{\prime} ; v^{\prime}:=v^{\prime}-t_{j-1, j} v_{j-1}$;

16. $t_{j, j}:=v_{j}^{*} v^{\prime} ; v^{\prime}:=v^{\prime}-t_{j, j} v_{j} ; t_{j+1, j}:=\left\|v^{\prime}\right\| ; v_{j+1}:=v^{\prime} / t_{j+1, j}$;

17. $\tau_{j}:=c_{j-1} t_{j, j}-s_{j-1} c_{j-2} t_{j-1, j}+p_{j}^{*} \hat{G}_{j,:}^{*}$;

18. $c_{j}:=\tau_{j} /\left(\left|\tau_{j}\right|^{2}+t_{j+1, j}^{2}\right)^{1 / 2} ; s_{j}:=t_{j+1, j} /\left(\left|\tau_{j}\right|^{2}+t_{j+1, j}^{2}\right)^{1 / 2} ; \gamma_{j}:=-s_{j} \gamma_{j-1}$;

19. $z_{j+1}:=-\left(v_{j}+t_{j, j} z_{j}+t_{j-1, j} z_{j-1}+W \hat{G}_{j,:}^{*}\right) / t_{j+1, j}$;

20. $x_{j}:=s_{j}^{2} x_{j-1}+\gamma_{j} c_{j}^{*} z_{j+1}$;

21. endfor

Iterations with GMRES are typically terminated when the residual vector (4.8) is sufficiently small, e.g., when

$$
\left\|r_{j}\right\| /\left\|r_{0}\right\| \leq \varepsilon
$$

for a user-specified value of $\varepsilon$. This stopping criterion can be easily evaluated, since Algorithm 4.4 computes $\gamma_{j}$, with $\left|\gamma_{j}\right|=\left\|r_{j}\right\|$, in each iteration. If the residual vectors are desired in each iteration, then one can add the relation (4.10) on line 10 (for $j=1$ ) and on line 20 of the algorithm. Stopping criteria of the type (4.18) have recently been discussed by Paige et al. $[13,14]$. In particular, the initial vector $x_{0}$ should be chosen so that $\left\|r_{0}\right\| \leq\|b\|$ and preferably as the zero-vector.

In order to make the connection between Algorithm 4.4 and the preceding discussion clearer, vectors are equipped with subscripts in the algorithm. However, only the most recently generated vectors $p_{j}^{*}$ and $x_{j}$ have to be stored simultaneously, and only the two most recently generated vectors $v_{j}, v_{j-1}$ and $z_{j}, z_{j-1}$ have to be stored at any given time. Only the $j$ th rows of the matrices $\hat{F}$ and $\hat{G}$ have to be stored simultaneously. The matrices $\tilde{F}$ and $W$ have to be stored and require $n \times s$ storage locations each. Moreover, representations of the matrices $A, F$, and $G$ have to be stored. Ignoring the storage for the latter, the storage requirement for Algorithm 4.4 is bounded by $(2 s+6) n+\mathcal{O}(s j)$ storage locations. The computational work per iteration is bounded independent of $j$; it is $\mathcal{O}(n)$ flops in addition to the arithmetic work required for the evaluation of $A v_{j}$. In the special case when $s=0$, Algorithm 4.4 simplifies to a minimal residual method for the solution of linear systems of equations with a symmetric, possibly indefinite, matrix.

We conclude this section with a comment on FOM, an iterative method that is closely related to GMRES; see Saad [15, section 6.4]. The $j$ th iterate determined by 
$\mathrm{FOM}, x_{j}^{\mathrm{FOM}} \in x_{0}+\mathcal{K}_{j}\left(A, r_{0}\right)$, satisfies

$$
b-A x_{j}^{\mathrm{FOM}} \perp \mathcal{K}_{j}\left(A, r_{0}\right) .
$$

From, e.g., [15, section 6.5.5] we know that the iterate $x_{j}^{\mathrm{FOM}}$ exists if and only if $\left|s_{j}\right|=\left\|r_{j-1}\right\| /\left\|r_{j}\right\|<1$, which is equivalent to $c_{j} \neq 0$, where $s_{j}$ and $c_{j}$ are entries of the Givens rotation $\Omega_{j+1}$; see (4.4). In this case, the relation between $x_{j}^{\mathrm{FOM}}$ and the GMRES iterate $x_{j}$ is given by

$$
x_{j}=s_{j}^{2} x_{j-1}+\left(1-s_{j}^{2}\right) x_{j}^{\mathrm{FOM}}
$$

see Saad [15, section 6.5.5] for details. A comparison with (4.10) shows that

$$
x_{j}^{\mathrm{FOM}}=\frac{\gamma_{j}}{c_{j}} z_{j+1},
$$

i.e., the vectors $z_{j+1}$ are FOM iterates up to normalization.

5. Computed examples. Linear systems of equations (1.2) with matrices of the form

$$
A=\left[\begin{array}{cc}
A_{1,1} & A_{1,2} \\
A_{2,1} & A_{2,2}
\end{array}\right] \in \mathbb{R}^{n \times n}
$$

with a symmetric leading principal submatrix $A_{1,1} \in \mathbb{R}^{(n-\ell) \times(n-\ell)}$ and $A_{1,2}, A_{2,1}^{*} \in$ $\mathbb{R}^{(n-\ell) \times \ell}, A_{2,2} \in \mathbb{R}^{\ell \times \ell}$, arise in many applications. Example 5.1 outlines a path following method that gives rise to matrices of this kind, and Examples 5.2-5.4 discuss the solution of integral equations. All computations were carried out in MATLAB with machine epsilon about $2 \cdot 10^{-16}$.

Example 5.1. We are interested in computing the solution $u$ of the nonlinear boundary value problem

$$
\begin{aligned}
-\Delta u-\lambda \exp (u) & =0 \quad \text { in } \quad S, \\
u & =0 \quad \text { on } \quad \partial S
\end{aligned}
$$

as a function of the parameter $\lambda$, where $\Delta$ denotes the Laplacian, $S$ the unit square, and $\partial S$ its boundary. This problem is known as the Bratu problem and is a common test problem for path following methods. We discretize $S$ by a uniform grid with $(\ell-1)^{2}$ interior grid points $\left(s_{k}, t_{k}\right)$, where $t_{k}=s_{k}=k / \ell, 1 \leq k<\ell$, and approximate the Laplacian by the standard five-point stencil. This yields a system of $(\ell-1)^{2}$ nonlinear equations

$$
G(w, \lambda)=0
$$

where the entries of the vector $w \in \mathbb{R}^{(\ell-1)^{2}}$ are approximations of the function $u$ at the grid points. Numerous techniques for computing $w(\lambda)$ as $\lambda$ is increased from, say, $\lambda_{0}$ to $\lambda_{1}$ are available; see, e.g., $[1,5,6]$ and the references therein.

The matrix $\partial G / \partial w$ is singular at turning points $(w, \lambda)$ of the path $\lambda \rightarrow(w(\lambda), \lambda)$, and one often introduces an auxiliary parameter $\eta$ in order to be able to traverse these points. Thus, let $\lambda=\lambda(\eta)$ and assume that $w(\lambda(\hat{\eta}))$ is available, where 
$\lambda_{0} \leq \lambda(\hat{\eta}) \leq \lambda_{1}$. We would like to determine $\lambda(\hat{\eta}+\delta \eta)$ and $w(\lambda(\hat{\eta}+\delta \eta))$. Introduce the function

$$
L(w, \lambda, \delta \eta)=d^{*}(w-w(\lambda(\hat{\eta})))+c(\lambda-\lambda(\hat{\eta}))-\delta \eta
$$

for some $d \in \mathbb{R}^{(\ell-1)^{2}}$ and $c \in \mathbb{R}$. The choice of $d$ and $c$ will be commented on below. Let $\left(w^{(j)}, \lambda^{(j)}\right)$ be an available approximation of the solution of

$$
\begin{aligned}
G(w, \lambda) & =0, \\
L(w, \lambda, \delta \eta) & =0 .
\end{aligned}
$$

Newton's method can be used to determine an improved approximation

$$
\left(w^{(j+1)}, \lambda^{(j+1)}\right)=\left(w^{(j)}+\delta w, \lambda^{(j)}+\delta \lambda\right)
$$

of the solution $(w(\lambda(\hat{\eta}+\delta \eta)), \lambda(\hat{\eta}+\delta \eta))$ of (5.5), where $\delta w$ and $\delta \lambda$ satisfy

$$
\left[\begin{array}{cc}
G_{w}^{(j)} & G_{\lambda}^{(j)} \\
d^{*} & c
\end{array}\right]\left[\begin{array}{c}
\delta w \\
\delta \lambda
\end{array}\right]=\left[\begin{array}{c}
-G^{(j)} \\
-L^{(j)}
\end{array}\right],
$$

with

$$
\begin{array}{ll}
G^{(j)}=G\left(w^{(j)}, \lambda^{(j)}\right), & L^{(j)}=L\left(w^{(j)}, \lambda^{(j)}, \delta \eta\right), \\
G_{w}^{(j)}=\frac{\partial}{\partial w} G\left(w^{(j)}, \lambda^{(j)}\right), & G_{\lambda}^{(j)}=\frac{\partial}{\partial \lambda} G\left(w^{(j)}, \lambda^{(j)}\right) .
\end{array}
$$

The vector $d$ should be chosen to make the matrix in (5.6) nonsingular even when $G_{w}$ is singular. This allows simple turning points to be traversed. The parameter $\eta$ is sometimes chosen to be arc length or pseudo-arc length of the curve $\lambda \rightarrow(w(\lambda), \lambda)$. The quantities $d, c$ in (5.4) then may be defined by, e.g., $d=d w(\lambda(\hat{\eta})) / d \eta, c=d \lambda(\hat{\eta}) / d \eta$.

To illustrate the performance of Algorithm 4.4, we discretize (5.1) on a uniform grid with $\ell=26$. The matrix in (5.6) then is of size $626 \times 626$. We choose $\lambda=\exp (\eta)-1$ and seek to determine the solution of (5.5) with $\delta \eta=10$, starting with $w^{(0)}=0$ and $\lambda^{(0)}=0$, i.e., $x_{0}=0$ in Algorithm 4.4. Then $G_{w}^{(0)}$ is the negative discrete Laplacian, $G_{\lambda}^{(0)}=-[1,1, \ldots, 1]^{*}, G^{(0)}=0$, and $L^{(0)}=-\delta \eta$. We let $c=1$ and, since $\partial w / \partial \eta$ is the largest at the center of the unit square, we choose $d=e_{(\ell-1)^{2} / 2}$. This defines the matrix in (5.6), which we will refer to as $A$. It has skew-symmetric part of rank $s=2$; cf. (1.1). We choose

$$
f_{1}=[1,1, \ldots, 1,0]^{*}-e_{(\ell-1)^{2} / 2}, \quad f_{2}=e_{(\ell-1)^{2}+1}, \quad g_{1}=f_{2}, \quad g_{2}=-f_{1}
$$

in the computations.

Algorithm 4.4 reduces the residual error from $10(=|\delta \eta|)$ to $1.84 \cdot 10^{-7}$ in 50 iterations. In the present example, the numerical values of $\left\|b-A x_{50}\right\|,\left\|r_{50}\right\|$ as computed by (4.10), and $\left|\gamma_{50}\right|$ agree to at least five significant digits. Solution of (5.6) by a direct method gave $x_{\text {direct }}$ with $\left\|x_{\text {direct }}-x_{50}\right\|=1.42 \cdot 10^{-10}$. Let $x_{50}^{\prime}$ denote the approximate solution determined by standard GMRES, ${ }^{1}$ and let $r_{50}^{\prime}=b-A x_{50}^{\prime}$. Then $\left\|r_{50}^{\prime}\right\|=1.84 \cdot 10^{-7},\left\|x_{\text {direct }}-x_{50}^{\prime}\right\|=1.42 \cdot 10^{-10}$, and $\left\|x_{50}^{\prime}-x_{50}\right\|=4.90 \cdot 10^{-12}$.

\footnotetext{
${ }^{1}$ Standard GMRES refers to the commonly used GMRES implementation based on the Arnoldi process with orthogonalization of the Arnoldi vectors by the modified Gram-Schmidt method.
}

Copyright $@$ ㅇ by SIAM. Unauthorized reproduction of this article is prohibited. 


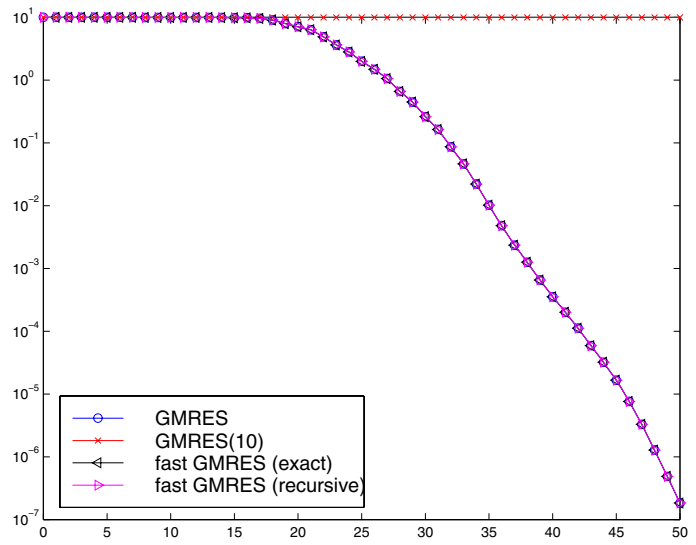

FIG. 5.1. Residual norms for Algorithm 4.4 applied to the data of Example 5.1. For comparison, we show both the norm of the exact residuals $\left\|b-A x_{k}\right\|$ (symbol $\triangleleft$ ) and the recursively computed residual norms $\left|\gamma_{k}\right|$ (symbol $\triangleright$ ), as well as the norm of the residuals $r_{k}^{\prime}$ (symbol $\circ$ ) obtained by standard GMRES, which are all of the same size. In contrast, restarted GMRES(10) (symbol $\times$ ) fails to converge.

Figure 5.1 shows the residual errors for standard GMRES and Algorithm 4.4. Let $x_{k}$ denote the iterates computed by Algorithm 4.4 and let $\gamma_{k}$ be the recursively evaluated quantities in the algorithm, such that (in exact arithmetic) $\left|\gamma_{k}\right|=\left\|b-A x_{k}\right\|$. Figure 5.1 displays $\left|\gamma_{k}\right|$, referred to as fast GMRES (recursive), as well as the evaluated norms $\left\|b-A x_{k}\right\|$, referred to as fast GMRES (exact), for $0 \leq k \leq 50$. The $\left|\gamma_{k}\right|$ are seen to be accurate approximations of $\left\|b-A x_{k}\right\|$. Moreover, the latter quantities are of the same size as the residual norms produced by standard GMRES.

Convergence is slow during the first 15 iterations and can be sped up by the use of a preconditioner. Note that a symmetric positive definite preconditioner would not change the rank of the skew-symmetric part.

Algorithm 4.4 requires about the same computer storage as GMRES restarted every $2 s+6$ iterations. The latter method is referred to as restarted GMRES $(2 s+6)$. We also compare Algorithm 4.4 to restarted GMRES $(2 s+6)$. For the present example restarted GMRES $(2 s+6)$ with $s=2$ fails to converge; see Figure 5.1.

Both standard and restarted GMRES are implemented using modified GramSchmidt orthogonalization of the Arnoldi vectors. Algorithm 4.4 explicitly orthogonalizes each new Arnoldi vector $v_{k+1}$ only against the two most recently generated vectors, $v_{k}$ and $v_{k-1}$. Therefore, the orthogonality properties of the matrices $V_{k}=\left[v_{1}, v_{2}, \ldots, v_{k}\right]$ determined by standard GMRES and Algorithm 4.4 in finite precision arithmetic may differ. Figure 5.2 displays the quantities $\left\|I_{k}-V_{k}^{*} V_{k}\right\|^{2}$, for $1 \leq k \leq 50$, for matrices $V_{k}$ determined by standard GMRES and Algorithm 4.4. In this example, the columns of the matrices $V_{k}$ determined by Algorithm 4.4 are closer to orthonormal than those determined by standard GMRES.

Example 5.2. The integral equation

$$
\gamma u(\alpha)+\frac{1}{\pi} \int_{-1}^{1} \frac{d}{d^{2}+(\alpha-\beta)^{2}} u(\beta) d \beta=f(\alpha), \quad-1 \leq \alpha \leq 1,
$$

with $\gamma=1$ and $d$ a positive constant, is known as Love's integral equation. It arises in electrostatics; see, e.g., Baker [3, p. 258]. Let $f(\alpha)=(1+\alpha)^{1 / 2}$, let $d=1 / 10$, and discretize (5.7) by a Nyström method based on the composite trapezoidal rule with 


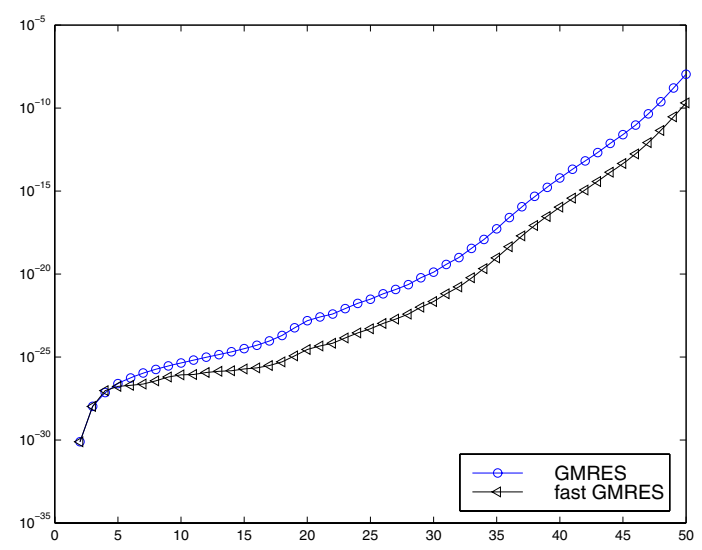

FIG. 5.2. Orthonormality of the Arnoldi vectors for Example 5.1: $\left\|I_{k}-V_{k}^{*} V_{k}\right\|^{2}$ as a function of $k$ for Algorithm 4.4 (symbol $\triangleleft$ ) and standard GMRES (symbol ०).

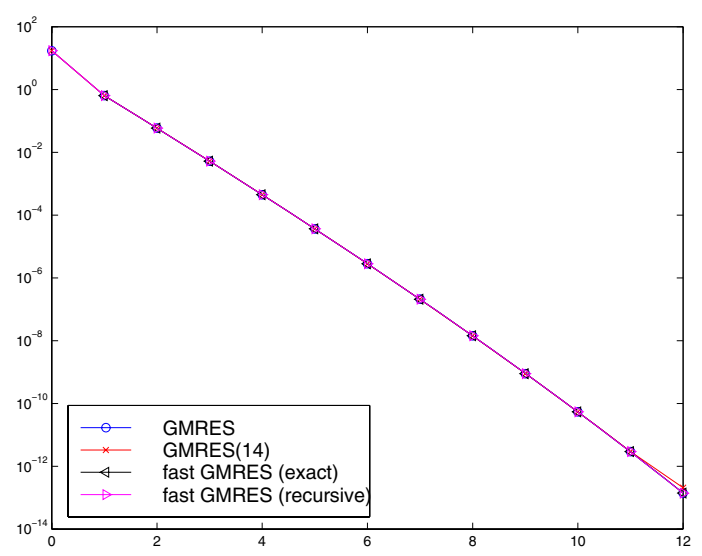

FIG. 5.3. Residual norms for Algorithm 4.4 applied to the data of Example 5.2. For comparison, we show both the norm of the exact residuals $\left\|b-A x_{k}\right\|$ (symbol $\triangleleft$ ) and the recursive residual norms $\left|\gamma_{k}\right|($ symbol $\triangleright)$, which are of the same size. The norm of the residuals $r_{k}^{\prime}$ obtained by standard GMRES (symbol $\circ)$ and by restarted GMRES(14) (symbol $\times$ ) are also displayed.

equidistant nodes $\alpha_{k}=\beta_{k}=(k-1) /(n-1), 1 \leq k \leq n, n=300$. This gives a linear system of equations with a matrix of the form

$$
A=\gamma I+K D
$$

where $K$ is a symmetric Toeplitz matrix and $D=\operatorname{diag}[1 / 2,1,1, \ldots, 1,1 / 2]$. The skew-symmetric part of $A$ therefore is of rank $s=4$. The memory requirement of Algorithm 4.4 is about the same as for restarted GMRES(14).

Figure 5.3 shows the residual errors for Algorithm 4.4 as given by $\left|\gamma_{k}\right|$ and $\left\|b-A x_{k}\right\|$ for $0 \leq k \leq 12$, as well as the corresponding residual errors for standard GMRES. The initial approximate solution is $x_{0}=0$. The iterations are terminated as soon as the residual error for standard GMRES is of norm smaller than $1 \cdot 10^{-12}$. Convergence is rapid both for Algorithm 4.4 and standard GMRES, and the methods produce iterates with residual errors of nearly the same size.

Copyright $\odot$ by SIAM. Unauthorized reproduction of this article is prohibited. 


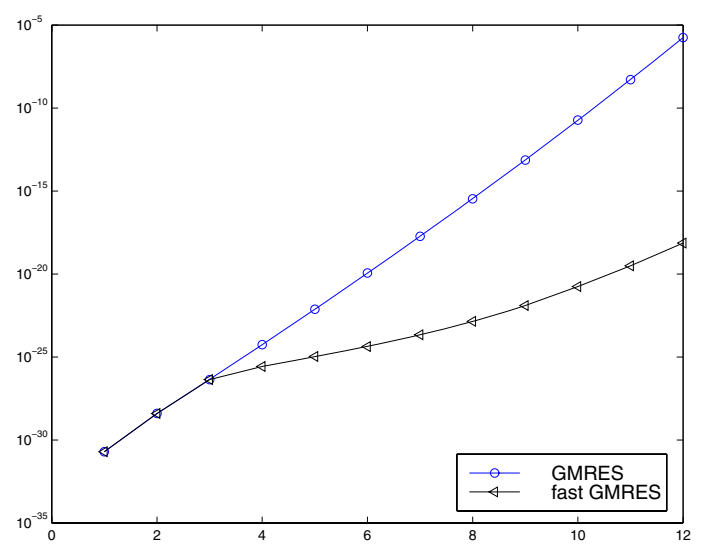

FIG. 5.4. Orthonormality of the Arnoldi vectors for Example 5.2: $\left\|I_{k}-V_{k}^{*} V_{k}\right\|^{2}$ as a function of $k$ for Algorithm 4.4 (symbol $\triangleleft$ ) and standard GMRES (symbol ०).

Figure 5.4 is analogous to Figure 5.2 and shows that the Arnoldi vectors generated by Algorithm 4.4 are slightly closer to being orthonormal than the Arnoldi vectors determined by standard GMRES.

The nonsymmetric matrix $K D$ in (5.8) is the discretization of a compact integral operator. It has many eigenvalues close to the origin. Therefore the matrix (5.8) has many eigenvalues close to $\gamma$, which has the value one in Example 5.2. In the following examples, we will reduce $\gamma$. This reduces the rate of convergence and illustrates that, differently from Examples 5.1 and 5.2, the Arnoldi vectors determined by Algorithm 4.4 may be less close to orthonormal than the Arnoldi vectors determined by the Arnoldi process in the standard GMRES implementation.

Example 5.3. We modify the integral equation (5.7) of Example 5.2 by setting $\gamma=0.1$. This change of $\gamma$ reduces the rate of convergence. Discretization is carried out in the same manner as in Example 5.2. We use the same initial approximate solution and stopping criterion as in Example 5.2.

Figure 5.5 displays the norm of the residual errors for Algorithm 4.4, standard GMRES, and restarted GMRES(14) and is analogous to Figure 5.3. Figure 5.5 shows the residual errors $r_{21}$ and $r_{22}$ determined by Algorithm 4.4 to be of slightly larger norm than the corresponding residual errors determined by standard GMRES. The cause for this can be found in Figure 5.6(a), which shows the quantities $\left\|I_{k}-V_{k}^{*} V_{k}\right\|^{2}$ for $1 \leq k \leq 22$. The figure shows the Arnoldi vectors computed by Algorithm 4.4 to be slightly less close to orthonormal than are the Arnoldi vectors determined by standard GMRES.

Figure 5.6(b) displays $\left\|I_{m+1}-V_{m-k: k}^{*} V_{m-k: k}\right\|^{2}$ as a function of $k$ for $m=$ $1,2, \ldots, 5$, thus measuring the orthonormality between the last $m+1$ Arnoldi vectors computed by Algorithm 4.4. Orthonormality is lost fairly rapidly for $m \geq 3$.

Example 5.4. We modify the integral equation (5.7) of Examples 5.2 and 5.3 by setting $\gamma=0.01$. This change of $\gamma$ reduces the rate of convergence compared with Example 5.3. Discretization is carried out in the same manner as in Examples 5.2 and 5.3, and we use the same initial approximate solution and stopping criterion as in those examples.

Figure 5.7 displays the norm of the residual errors for Algorithm 4.4, standard GMRES, and restarted GMRES(14) and is analogous to Figure 5.5. Figure 5.7 shows 


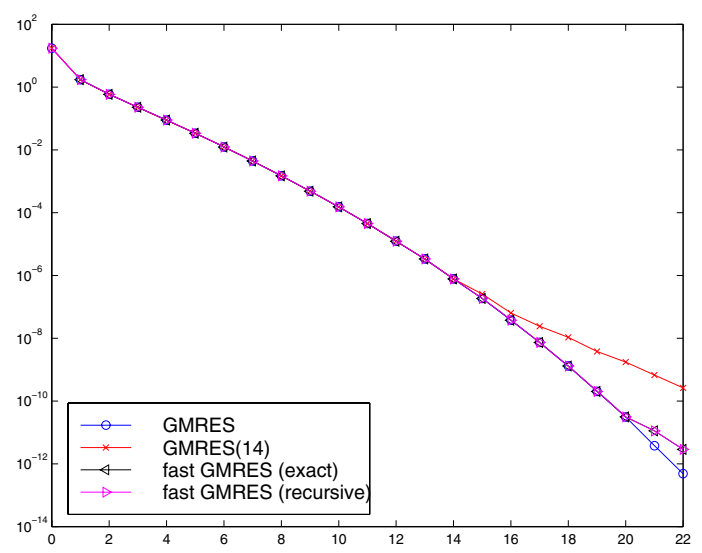

FIG. 5.5. Residual norms for Algorithm 4.4 applied to the data of Example 5.3. For comparison, we display both the norm of the exact residuals $\left\|b-A x_{k}\right\|$ (symbol $\triangleleft$ ) and the recursive residual norms $\left|\gamma_{k}\right|$ (symbol $\left.\triangleright\right)$ ), which are of the same size, and slightly smaller than those obtained for restarted GMRES(14) (symbol $\times$ ). The norms of the residuals $r_{k}^{\prime}$ determined by standard GMRES (symbol $\circ$ ) are somewhat smaller for $k \geq 21$.

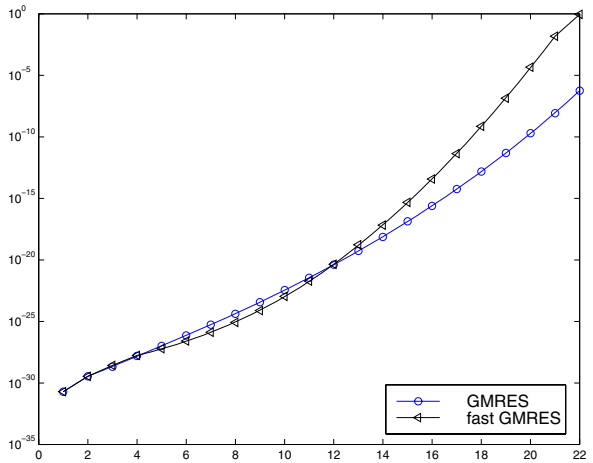

(a)

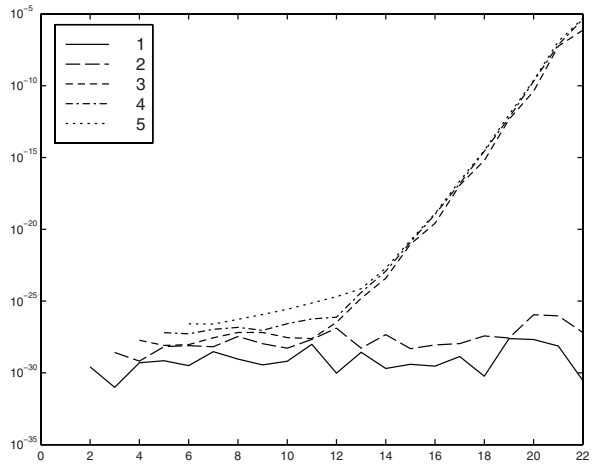

(b)

FIG. 5.6. Orthonormality of the Arnoldi vectors for Example 5.3: (a) $\left\|I_{k}-V_{k}^{*} V_{k}\right\|^{2}$ as a function of $k$ for Algorithm 4.4 (symbol $\triangleleft$ ) and standard GMRES (symbol o). (b) From bottom to top, $\left\|I_{m+1}-V_{m-k: k}^{*} V_{m-k: k}\right\|^{2}$ as a function of $k$ for $m=1,2, \ldots, 5$ for Algorithm 4.4 .

Algorithm 4.4 to reduce the norm of the residual error slower than standard GMRES, but faster than restarted GMRES(14).

The reason for the slower convergence of Algorithm 4.4 is the loss of orthonormality of the Arnoldi vectors generated by the algorithm. The latter is illustrated by Figures 5.8.

Examples 5.3 and 5.4 illustrate that the iterates determined by Algorithm 4.4 may converge slower to the solution than the iterates determined by standard GMRES. A reason for this appears to be that the Arnoldi vectors generated by Algorithm 4.4 may be far from orthonormal; see Example 5.4. The loss of orthogonality and its effect on the convergence of GMRES has received considerable attention in the literature; see, e.g., $[8,10,13,14,16,17]$. For instance, Simoncini and Szyld [16] recently pointed out that loss of orthogonality does not prevent a near-optimal rate of convergence, 


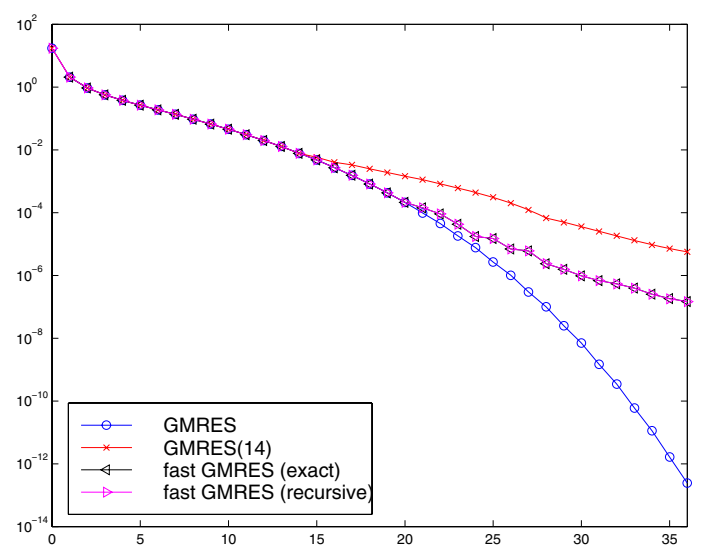

FIG. 5.7. Residual norms for Algorithm 4.4 applied to the data of Example 5.3. For comparison, we show both the norm of the exact residuals $\left\|b-A x_{k}\right\|$ (symbol $\triangleleft$ ) and the recursive residual norms $\left|\gamma_{k}\right|$ (symbol $\left.\triangleright\right)$, which are of the same size, and smaller than those obtained by restarted GMRES(14) $($ symbol $\times)$. The norms of the residuals $r_{k}^{\prime}$ obtained by the standard GMRES (symbol $\circ$ ) are much smaller for $k \geq 30$.

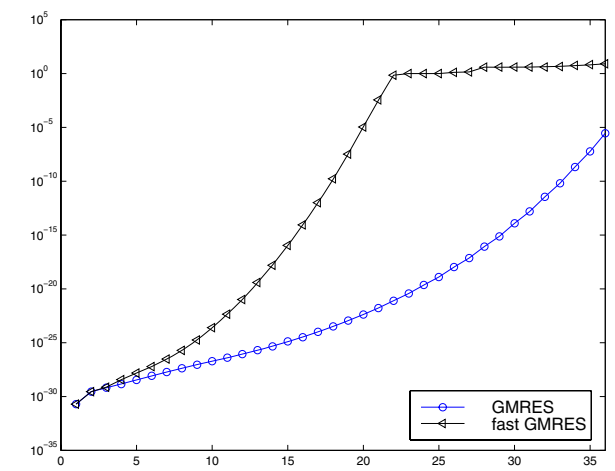

(a)

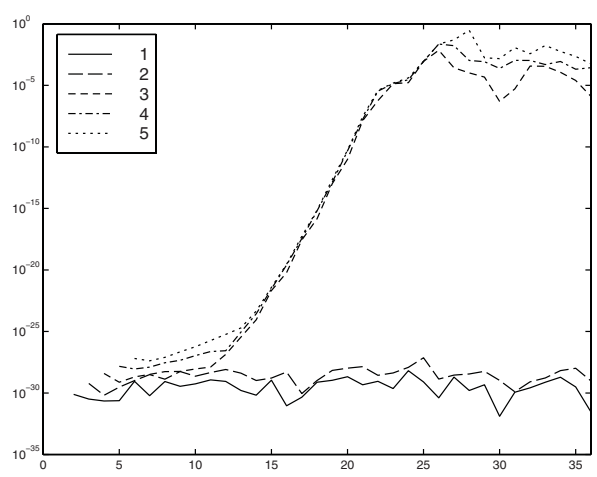

(b)

FIG. 5.8. Orthonormality of the Arnoldi vectors for Example 5.3: (a) $\left\|I_{k}-V_{k}^{*} V_{k}\right\|^{2}$ as a function of $k$ for Algorithm 4.4 (symbol $\triangleleft$ ) and standard GMRES (symbol ०). (b) From bottom to top, $\left\|I_{m+1}-V_{m-k: k}^{*} V_{m-k: k}\right\|^{2}$ as a function of $k$ for $m=1,2, \ldots, 5$ for Algorithm 4.4.

provided that each new Arnoldi vector generated has a sufficiently large angle with the space spanned by the already available Arnoldi vectors. Example 5.4 suggests that the loss of orthogonality also may reduce this angle.

6. Conclusion. Linear systems of equations with a matrix that satisfies (1.1) with a small value of $s$ arise in a variety of applications. For many, but not all, linear systems of equations of this kind, Algorithm 4.4 converges like standard GMRES, but requires less computer storage and arithmetic work. In all our experiments, Algorithm 4.4 converges faster than restarted GMRES $(2 s+6)$, which demands roughly the same amount of computer storage as Algorithm 4.4.

Acknowledgment. We would like to thank a referee for comments. 


\section{REFERENCES}

[1] E. L. Allgower and K. Georg, Numerical Continuation Methods, Springer, Berlin, 1990.

[2] G. Arnold, N. Cundy, J. van den Eshof, A. Frommer, S. Krieg, Th. Lippert, and K. SCHÄFER, Numerical methods for the QCD overlap operator II: Optimal Krylov subspace methods, in QCD and Numerical Analysis III, A. Boricci, A. Frommer, B. Joó, A. Kennedy, and B. Pendleton, eds., Lect. Notes Comput. Sci. Eng. 47, Springer, Berlin, 2005, pp. 153-167.

[3] C. T. H. BAKer, Numerical Treatment of Integral Equations, Clarendon Press, Oxford, 1977.

[4] T. Barth And T. Manteuffel, Multiple recursion conjugate gradient algorithms, part I: Sufficient conditions, SIAM J. Matrix Anal. Appl., 21 (2000), pp. 768-796.

[5] D. Calvetti And L. Reichel, Iterative methods for large continuation problems, J. Comput. Appl. Math., 123 (2001), pp. 217-240.

[6] C.-S. Chen, N.-H. Lu, And Z.-L. Weng, Conjugate gradient methods for continuation problems II, J. Comput. Appl. Math., 62 (1995), pp. 197-216.

[7] P. Concus And G. H. Golub, A generalized conjugate gradient method for nonsymmetric systems of linear equations, in Computing Methods in Applied Science and Engineering, R. Glowinski and J. L. Lions, eds., Springer, New York, 1976, pp. 56-65.

[8] J. Drkosova, A. Greenbaum, M. Rozloznik, and Z. Strakos, Numerical stability of GMRES, BIT, 35 (1995), pp. 309-330.

[9] Yu. Eidelman, I. Gohberg, and V. Olshevsky, The QR iteration method for Hermitian quasiseparable matrices of an arbitrary order, Linear Algebra Appl., 404 (2005), pp. 305-324.

[10] A. Greenbaum, M. Rozloznik, and Z. Strakos, Numerical behavior of the modified GramSchmidt GMRES implementation, BIT, 37 (1997), pp. 706-719.

[11] C. Jagels And L. Reichel, The isometric Arnoldi process and an application to iterative solution of large linear systems, in Iterative Methods in Linear Algebra, R. Beauwens and P. de Groen, eds., Elsevier, Amsterdam, 1992, pp. 361-369.

[12] C. Jagels And L. Reichel, A fast minimal residual algorithm for shifted unitary matrices, Numer. Linear Algebra Appl., 1 (1994), pp. 555-570.

[13] C. C. Paige, M. Rozložník, and Z. Strakoš, Modified Gram-Schmidt (MGS), least squares, and backward stability of MGS-GMRES, SIAM J. Matrix Anal. Appl., 28 (2006), pp. 264-284.

[14] C. C. Paige And Z. Strakoš, Residual and backward error bounds in minimum residual Krylov subspace methods, SIAM J. Sci. Comput., 23 (2002), pp. 1898-1923.

[15] Y. SAAD, Iterative Methods for Sparse Linear Systems, 2nd ed., SIAM, Philadelphia, 2003.

[16] V. Simoncini And D. B. SzYLD, The effect of non-optimal bases on the convergence of Krylov subspace methods, Numer. Math., 100 (2005), pp. 711-733.

[17] Z. Strakoš And J. Liesen, On numerical stability in large scale linear algebraic computations, Z. Angew. Math. Mech., 85 (2005), pp. 307-325.

[18] O. WidLund, A Lanczos method for a class of nonsymmetric systems of linear equations, SIAM J. Numer. Anal., 15 (1978), pp. 801-812.

Copyright (c) by SIAM. Unauthorized reproduction of this article is prohibited. 